\title{
Efficacité biologique des herbicides ACTION $80 ®$ et LUMAX® dans le contrôle des adventices dans les pépinières sèches de Tectona grandis à Sangoué en Côte d'Ivoire
}

${ }^{1}$ KONE Cédessia Hervé Kéassemon, ${ }^{1}$ YAPI Arnaud-Freddy, ${ }^{2}$ GUE Arsène.

'Université Peleforo GON COULIBALY, UFR des Sciences Biologiques, Département de Biologie Végétale, BP 1328 Korhogo, Côte d'lvoire.

${ }^{2}$ Université Jean Lorougnon Guédé, UFR Agroforesterie, Laboratoire d'Amélioration de la production agricole, BP 150 Daloa, Côte d'Ivoire.

E-mail address of corresponding author : yapi.arnaudfreddy@gmail.com

Original submitted in on $2^{\text {nd }}$ July 2020. Published online at www.m.elewa.org/journals/ on $30^{\text {th }}$ September 2020 https://doi.org/10.35759/JABs.153.3

\section{RESUME :}

Objectif: Deux herbicides "Action 80®» de matière active le Diuron (3-(3,4-Dichlorophenyl)-1,1Dimethylurea) à $80 \%$ et « Lumax ${ }^{\circledR}$ SE » de formulation ( $125 \mathrm{~g} / \mathrm{L}$ de Terbuthiron, $37,5 \mathrm{~g} / \mathrm{L}$ de Mesotrione et $375 \mathrm{~g} / \mathrm{L}$ de S-Métolachlore) ont été testés en vue d'évaluer l'efficacité biologique de chaque produit et sa dose minimale efficace sur le contrôle des adventices dans la culture du Teck.

Méthodologie et résultats : Pour pallier le déficit en main d'œuvre et résoudre le problème des adventices difficiles à maîtriser en entretien manuel, une série d'essais herbicides a été initiée dans les pépinières sèches de Tectona grandis L. dans la forêt classée de la Sangoué. Plusieurs doses des herbicides Lumax $®$ et Action $80 \circledR$ ont été testées en traitement de pré émergence des adventices.

Conclusion et application des résultats : Ces herbicides ont montré une bonne efficacité, la persistance des traitements varie entre 4 et 6 mois. Par ailleurs, la dose minimale efficace est de l'ordre de $3 \mathrm{l} / \mathrm{ha}$ pour Lumax囚 et de $4 \mathrm{~kg} /$ ha pour l'Action $80 \AA$.

Mots clés : adventices, contrôle chimique, reboisement, Teck, pépinières sèches

Biological efficacy of ACTION® and LUMAX® herbicides in weed control in dry nurseries of Tectona grandis in Sangoué, Côte d'Ivoire

ABSTRACT

Objective: Two herbicides, "Action 80®" with active ingredient Diuron (3- (3,4-Dichlorophenyl) -1,1Dimethylurea) at $80 \%$ and "Lumax® SE" of formulation (125 g/L of Terbuthiron, $37,5 \mathrm{~g} / \mathrm{L}$ of Mesotrione and $375 \mathrm{~g} / \mathrm{L}$ of S-Metolachlor) have been tested to assess the biological efficacy of each product and its minimum effective dose for weed control in Teak cultivation.

Methodology and results: To overcome the deficit of man power and resolve the weed problem difficult to control in manual maintenance, a series of herbicide trials was initiated in the dry nurseries of Tectona grandis $L$. in the classified forest of Sangoué. Several doses of the herbicides Lumax® and Action 80®were tested for weed pre-emergence treatment. 
Conclusion and application of results: These herbicides have shown good efficacy, the persistence of treatments varies between 4 and 6 months. In addition, the minimum effective dose is around $31 /$ ha for Lumax $\odot$ and $4 \mathrm{~kg} / \mathrm{ha}$ for Action $80 \AA$.

Key-words: Weeds, chemical control, reforestation, teak, dry nurseries.

\section{INTRODUCTION}

La reconstitution du capital forestier ivoirien a conduit à la mise en place d'une politique de reboisement intensif dans de nombreuses forêts classées de Côte d'Ivoire. A cet effet, de grandes plantations monospécifiques ont été créés après déboisement systématique à partir de 1966. Dès1984, la préparation mécanisée des parcelles a laissé place à une préparation manuelle. $\mathrm{Ce}$ changement de technique a induit la résurgence et la prolifération de certaines espèces difficilement contrôlables au cours des activités d'entretien des jeunes parcelles et du suivi sylvicole. Le teck, de nom scientifique Tectona grandis $\mathrm{L}$. est l'espèce de reboisement industrielle la plus prisée en Côte d'Ivoire avec une moyenne annuelle de 76000 ha en 2009. En effet, grâce à sa croissance rapide et sa couronne peu réduite dans son jeune âge, les parcelles sous culture de teck sont sujettes à des associations avec plusieurs cultures vivrières. Malheureusement, suite à la raréfaction et la cherté de la main d'œuvre, la gestion efficace de l'enherbement dans les plantations de teck est devenue pénible. Or, les adventices servent généralement d'hôtes ou réservoirs de déprédateurs et exercent des phénomènes d'allélopathie sur les plantes cultivées. Tous ces caractères impactent négativement le rendement et la qualité de la production des cultures (Koch et Beshir, 1982 ; Orkwor, 1983; Caussanel., 1989, Kouamé, 2014 ; Yapi, 2017). Face à ces difficultés, les producteurs, généralement peu nantis, sont amenés à utiliser des techniques souvent rudimentaires et peu adaptées. Selon Dore et al., (2006), les difficultés techniques sont les

\section{MATERIEL ET METHODES}

Matériel biologique : Il est constitué de semences de teck et de la diversité d'adventices présentes dans les pépinières sèches et humides.

Matériel technique: Le matériel technique est constitué essentiellement d'un pulvérisateur à dos à principales causes de la mauvaise maîtrise de la gestion de l'enherbement des cultures. Ces mauvaises techniques de contrôle des adventices sont à la base de la prolifération des bioagresseurs. Ainsi, la maîtrise des adventices devient-elle une préoccupation majeure pour les producteurs et la structure Etatique en charge du reboisement du teck (SODEFOR). Cette dernière dépense en moyenne $55000 \mathrm{~F} / \mathrm{ha} / \mathrm{an}$ pour l'entretien manuel des jeunes parcelles. Afin d'explorer d'autres voies la SODEFOR a relancé les essais herbicides qui avaient été abandonnés en 1984 (SODEFOR, 1998). En effet, Ndabalishye, (1995) et Yapi, (2017) ont montré que la lutte chimique peut réduire de façon significative le temps du désherbage et accroître de 10 à $20 \%$ la production par rapport aux techniques de désherbage manuel. Selon, Hamel (1986) l'usage des techniques de désherbages manuels dans les palmeraies revenaient plus coûteux que le contrôle chimique des adventices. La présente étude a été entreprise pour pallier le déficit de la main d'œuvre et réduire les coûts excessifs des désherbages manuels. Elle a pour objectif principal de tester l'efficacité biologique de deux herbicides "Lumax $®$ SE » et "Action $80 ®$ » sur les semences d'adventices et leur impact sur les graines de Tectona grandis dans les pépinières sèches. Spécifiquement, il est question de définir la dose minimale efficace de chaque herbicide, d'évaluer la persistance des herbicides dans le sol (rémanence de chaque produit) et de tester l'effet des doses d'herbicide sur la germination des semences de teck (phytotoxicité).

pression entretenue, d'un matériel d'inventaire et de confection d'herbier.

Produits chimiques: Ils sont constitués des herbicides Action $80 \circledR$ WP et Lumax® SE. Action $80 \circledR$ WP est un herbicide utilisé en préémergence des 
adventices. Sa matière active est le Diuron (3-(3,4Dichlorophenyl)-1,1-Dimethylurea) à $80 \%$. Lumax® SE est issu de la combinaison de 3 matières actives de concentration respectives (125 g/L de Terbuthiron, 37,5 $\mathrm{g} / \mathrm{L}$ de Mesotrione et $375 \mathrm{~g} / \mathrm{L}$ de S-Métolachlore. Tous ces deux produits sont homologués et vendus sur le marché ivoirien.

Applications herbicides: Quatre doses des herbicides de pré-émergence Action $80 \AA$ WP et Lumax® SE ont été retenus. Ces doses ont été comparées à un témoin non traité. Pour chaque dose, quatre (4) répétitions sont faites. Les doses utilisées par herbicide et la codification des traitements sont indiquées dans le tableau 1. Le volume de bouillie a été fixé à $600 \mathrm{~L} / \mathrm{h}$. Les essais d'herbicides ont été réalisés en plein champ.

Méthode d'évaluation de l'efficacité des traitements chimiques : L'efficacité biologique et la rémanence de chaque dose d'herbicide utilisé ont été faites à l'aide d'un comptage du nombre de plantules d'adventices ayant germé, du taux de recouvrement des parcelles et la rémanence des produits. Le taux de recouvrement de chaque parcelle par les adventices est estimé en pourcentage. Le dénombrement de plantules d'adventices et le taux recouvrement se sont faits de façon hebdomadaire. Cependant, leurs nombres moyens ont été comparés chaque mois pendant une période de six mois. Ces observations ont été faites afin d'apprécier l'effet des traitements chimiques (parcelles traitées) sur la flore d'adventices en comparaison à celle des parcelles témoins. De plus, un essai portant sur l'effet des herbicides sur les semences de teck et sur la sensibilité des espèces d'adventices a été réalisé. Cet essai a été également réalisé afin de confirmer ou infirmer l'efficacité des herbicides sur la germination des semences d'adventices. Les observations ont duré 90 jours. Au cours du temps, le traitement est dit toujours efficace lorsque le rapport entre la productivité (degré d'infestation) de la parcelle traitée et celle de la parcelle témoin est inférieur à $50 \%$. Au-delà de ce taux, l'effet du traitement n'est plus perceptible.

Analyse des données : Le logiciel EXCEL a permis de saisir les données collectées. Puis, à l'aide du logiciel STATISTICA version 7.1, les différentes analyses de variance ont été réalisées. La classification des traitements a été faite à l'aide des tests de Duncan et de Newman-Keuls.

Tableau 1 : Doses d'herbicides testées en pré-émergence des adventices

\begin{tabular}{c|c|c}
\hline Herbicides & DOSES en Kg/ha & Codes \\
\hline \multirow{3}{*}{ ACTION 80® } & 2,5 & Ac2, 5 \\
& 2 & Ac2 \\
& 1,5 & Ac1, 5 \\
& 1 & Ac1 \\
\hline \multirow{2}{*}{ LUMAX® } & 5 & Lu5 \\
& 4 & Lu4 \\
& 3 & Lu3 \\
& 2 & Lu2 \\
\hline
\end{tabular}

\section{RESULTATS}

Efficacité biologique des herbicides sur les semences d'adventices: Au terme de l'expérience (six (6) mois après épandage), 75820 plantules dont 3696 Monocotylédones et 72124 Dicotylédones ont été dénombrées sur une superficie de $24 \mathrm{~m}^{2}$. Le tableau 2 indique le nombre moyen de plantules levées au cours de l'expérience. Les analyses statistiques réalisées montrent des différences significatives au niveau du nombre moyen de plantules sur chaque parcelle traitée et celui des parcelles du témoin non traité. Un mois après épandage, le traitement le moins efficace est AC1 (Action $80 \circledR$ à $1 \mathrm{Kg} / \mathrm{ha}$ ) avec un nombre moyen de plantules égal à 77 plantules $/ \mathrm{m}^{2}$. A partir de deux mois jusqu'à trois mois après l'épandage des produits, les nombres de plantules germées sur chaque sont statistiquement identiques quel que soit la dose et l'herbicide utilisé. Jusqu'à six (6) mois après épandage, les nombres de plantules sont statistiquement identiques sur toutes les parcelles traitées avec l'herbicide Action $80 \AA$ et avec la dose de Lumax® à 5 $\mathrm{Kg} / \mathrm{ha}$ (LU5), à l'exception de la dose minimale AC1 du produit Action $80 \AA$. L'analyse de variance indique que les traitements appliqués sont statistiquement différents. Le test de comparaison des moyennes de Duncan au niveau de confiance $95 \%$ permet de distinguer trois classes. Les doses de traitements LU2, 
LU3, LU4 et AC1 constituent une classe homogène avec des parcelles ayant les nombres de plantules les plus élevés. Toutefois, ces nombres de plantules restent inférieurs à celui dénombré sur les parcelles non traitées. Au niveau des taux d'enherbement, c'est après un mois d'épandage des produits que l'on observe une superficie importante recouverte par les adventices. Cependant, le plus faible taux moyen de recouvrement observé avec le traitement $\mathrm{AC} 1$ avec une productivité semencière estimée à $55,80 \%$ est supérieur au seuil d'efficacité de $50 \%$ (Tableau 3). Toutefois, il y'a différence entre les efficacités de produits en fonction de la dose du produit et du moment d'observation. Les résultats font distinguer trois (3) classes de traitements en fonction de la durée de la rémanence de ceux-ci. La dose de traitement «LU2 » constitue la dose à faible durée de rémanence (inférieure à deux mois). Le second groupe renferme les traitements LU3 et LU4. Ces doses de traitements ont une rémanence inférieure à quatre (4) mois. Enfin, le dernier groupe renfermant les traitements à longue rémanence est composé des traitements LU5, AC2.5, $A C 2$ et AC1.5. Leur persistance est supérieure à six (6) mois. La sensibilité des espèces majeures aux doses appliquées est donnée dans le tableau 4. L'analyse de ce tableau montre que la tolérance des adventices aux traitements ne suit pas une fonction linéaire relativement à l'augmentation de la dose de l'herbicide. Dans certains cas, l'augmentation de la dose n'influence aucunement la sensibilité de l'espèce. De façon générale, les adventices majeures sont plus sensibles lorsque les doses de produits sont élevées. Les espèces telles qu'Euphorbia heterophylla, Lantana camara et Mimosa invisa sont insensibles aux différentes doses de Lumax®. Tandis qu'Accoreras zizanioides, Chromolaena odorata, Merremia aegyptiaca, Panicum maximum, Physalis angulata, Physalis micrantha, Solanum verbascifolium et Periploca nigrescens montrent des sensibilités aux différentes doses de traitements.

Efficacité et rémanence des traitements appliqués : Au terme des 90 jours d'observations après l'épandage, 15 espèces et 14 genres ont été inventoriés sur le périmètre expérimental. Ces espèces se répartissent en 10 familles. Les espèces les plus représentées en nombre de plantules sur les placettes traitées avec Lumax® et Action $80 \AA$ sont Centrosema pubescens (Fabaceae) et Schrankia leptocarpa (Mimosaceae). Le tableau 5 donne les effectifs cumulés du nombre de plants qui ont effectivement germé en fonction du temps. L'analyse de variance indique que les traitements appliqués sont statistiquement différents. Le test de comparaison des moyennes de Duncan au niveau de confiance $95 \%$ permet de distinguer deux (2) classes de traitements à tous les niveaux de collectes des données. Les traitements appliqués constituent une seule classe et l'autre classe renferme le témoin non traité. Durant toute la période d'observation, le nombre moyen de plantules ayant germées par placette est toujours inférieur à 100 plantules. Le rapport entre la productivité (degré d'infestation) de la parcelle traitée et celle de la parcelle témoin est dressé dans le tableau 6. Le seuil d'enherbement critique étant de $50 \%$, les plus fortes doses de produits AC5 et LU5 ont montré une bonne efficacité. Leurs taux d'enherbement sont respectivement de $33,14 \%$ et $29,71 \%$ au terme des observations. Ces deux traitements sont les plus rémanents. Les doses AC2.5 et LU2.5 ont une rémanence à deux mois.

Effet des traitements sur les semences de Teck: L'évolution du nombre de graines germées est illustrée par la figure 1. Le comptage a été effectué pendant trois (3) mois. Selon la courbe d'évolution du taux moyen de germination, les taux les plus élevés sont atteints deux (2) mois après le semis. L'analyse de variance effectuée à cette date montre que le taux de germination varie statistiquement d'un traitement à un autre (Tableau 7). Le test de comparaison des moyennes de Duncan au niveau de confiance $95 \%$ permet de distinguer trois (3) classes de traitements (Figure 2). Ainsi, les traitements AC5 et LU2.5 offrentils les meilleurs taux de germination des semences de Teck, tandis que la germination de celles-ci a été inhibée par le traitement LU5. 


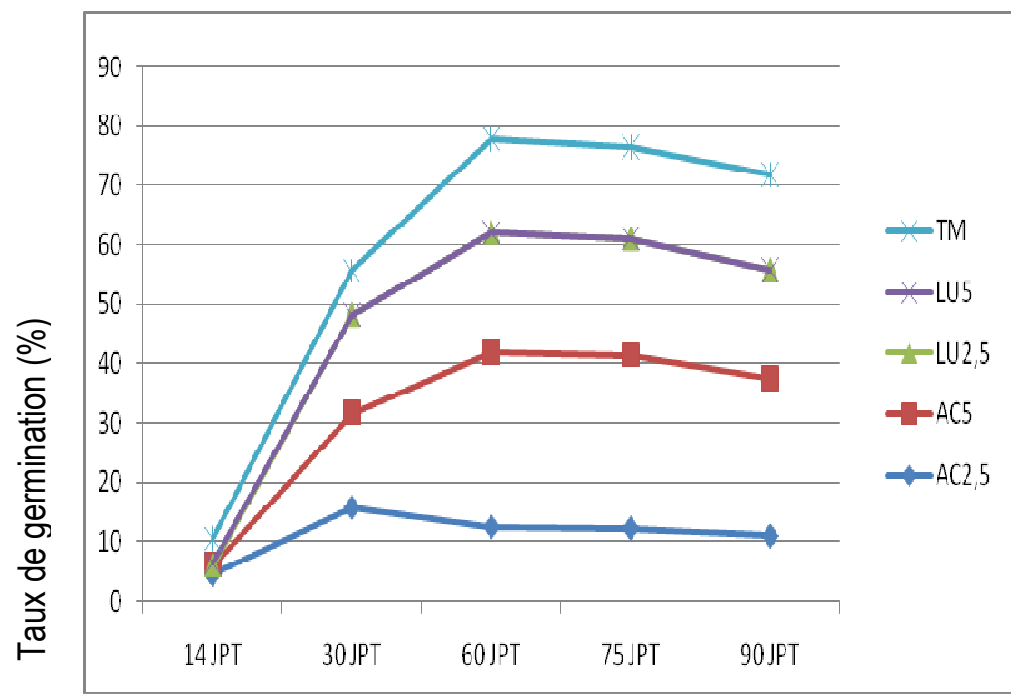

Temps

Figure 1 : Évolution du taux de germination des semences de Teck en fonction des traitements appliqués

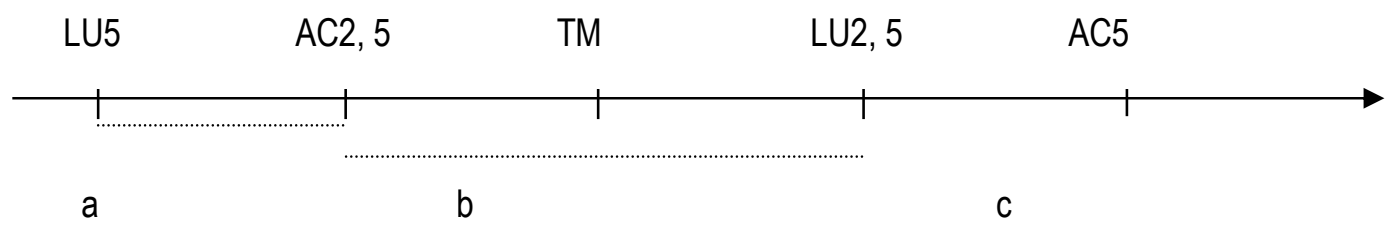

Figure 2 : Classement des traitements en fonction du taux de germination des semences de Teck, 2 mois après application des traitements

Tableau 2 : Nombre moyen de plantules germées au cours du temps

\begin{tabular}{l|c|c|c|c|c|c}
\hline Doses par ha & $\mathbf{1}$ mois & $\mathbf{2}$ mois & $\mathbf{3}$ mois & $\mathbf{4}$ mois & $\mathbf{5}$ mois & $\mathbf{6}$ mois \\
\hline AC2,5 & $22 a$ & $29 a$ & $60 a$ & $118 a$ & $174 a$ & $191 a$ \\
AC2 & $62 a$ & $73 a$ & $141 a$ & $187 a$ & $225 a$ & $231 a$ \\
AC1,5 & $48 a$ & $63 a$ & $94 a$ & $116 a$ & $138 a$ & $140 a$ \\
AC1 & $77 a b$ & $92 a$ & $123 a$ & $193 a$ & $268 a b$ & $290 a b$ \\
TM & $138 b$ & $184 b$ & $317 b$ & $415 b$ & $500 b$ & $504 b$ \\
LU5 & $4 a$ & $14 a$ & $92 a$ & $153 a$ & $198 a$ & $206 a$ \\
LU4 & $50 a$ & $63 a$ & $138 a$ & $246 a b$ & $307 a b$ & $320 a b$ \\
LU3 & $57 a$ & $80 a$ & $143 a$ & $212 a$ & $292 a b$ & $300 a b$ \\
LU2 & $68 a$ & $93 a$ & $172 a$ & $235 a b$ & $298 a b$ & $308 a b$ \\
\hline
\end{tabular}


Tableau 3 : Évolution du taux d'enherbement sur chaque placette traitée en fonction du temps

\begin{tabular}{c|c|c|c|c|c|c}
\hline Traitements & $\mathbf{1}$ mois & $\mathbf{2}$ mois & $\mathbf{3}$ mois & $\mathbf{4}$ mois & $\mathbf{5}$ mois & $\mathbf{6}$ mois \\
\hline AC2,5 & 15,94 & 15,76 & 18,93 & 28,43 & 34,80 & 37,90 \\
AC2 & 44,93 & 39,67 & 44,48 & 45,06 & 45,00 & 45,83 \\
AC1,5 & 34,78 & 34,24 & 29,65 & 27,95 & 27,60 & 27,78 \\
AC1 & 55,80 & 50,00 & 38,80 & 46,51 & 53,60 & 57,54 \\
LU5 & 2,90 & 7,61 & 29,02 & 36,87 & 39,60 & 40,87 \\
LU4 & 36,23 & 34,24 & 43,53 & $\mathbf{5 9 , 2 8}$ & $\mathbf{6 1 , 4 0}$ & $\mathbf{6 3 , 4 9}$ \\
LU3 & 41,30 & 43,48 & 45,11 & $\mathbf{5 1 , 0 8}$ & $\mathbf{5 8 , 4 0}$ & $\mathbf{5 9 , 5 2}$ \\
LU2 & 49,28 & $\mathbf{5 0 , 5 4}$ & $\mathbf{5 4 , 2 6}$ & $\mathbf{5 6 , 6 3}$ & $\mathbf{5 9 , 6 0}$ & $\mathbf{6 1 , 1 1}$ \\
\hline
\end{tabular}

Tableau 4 : Sensibilité des semences d'adventices aux traitements appliqués

\begin{tabular}{|c|c|c|c|c|c|c|c|c|c|}
\hline $\mathrm{N}^{\circ}$ & Espèces & AC2.5 & $\mathrm{AC2}$ & AC1.5 & AC1 & LU5 & |LU4 & LU3 & LU2 \\
\hline 1 & Accoreras zizanioides & ++ & ++ & ++ & ++ & ++ & ++ & ++ & ++ \\
\hline 2 & Centrosema pubescens & + & - & - & + & ++ & ++ & - & - \\
\hline 3 & Chromolaena odorata & ++ & ++ & ++ & ++ & ++ & + & + & + \\
\hline 4 & Croton hirtus & ++ & + & - & - & + & + & + & + \\
\hline 5 & Euphorbia heterophylla & + & - & + & - & - & - & - & - \\
\hline 6 & Euphorbia hyssopifolia & ++ & + & - & - & + & + & + & + \\
\hline 7 & Lantana camara & ++ & - & ++ & ++ & - & - & - & - \\
\hline 8 & Merremia aegyptiaca & ++ & - & ++ & ++ & ++ & ++ & ++ & - \\
\hline 9 & Mimosa invisa & - & - & + & - & - & - & - & - \\
\hline 10 & Panicum maximum & + & + & + & - & ++ & ++ & + & + \\
\hline 11 & Periploca nigrescens & ++ & ++ & ++ & - & ++ & ++ & ++ & ++ \\
\hline 12 & Physalis angulata & ++ & t+ & t+ & ++ & ++ & + & + & - \\
\hline 13 & Physalis micrantha & ++ & ++ & ++ & ++ & ++ & ++ & ++ & ++ \\
\hline 14 & Portulaca quadrifida & + & + & + & + & + & + & + & + \\
\hline 15 & Solanum verbascifolium & ++ & ++ & ++ & ++ & + & + & + & + \\
\hline
\end{tabular}

++ : Espèce sensible $\quad+$ : Espèce peu sensible $\quad-:$ : Espèce insensible

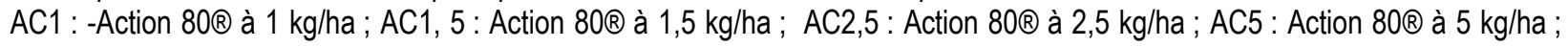

LU2 : Lumax® à 2L /ha ; LU3 : Lumax® à 3 L/ha ; LU4 : Lumax®à 4 L/ha ; LU5 : Lumax® à 5 L/ha

Tableau 5 : Évolution du nombre moyen de plantules en fonction des traitements

\begin{tabular}{c|c|c|c|c}
\hline Traitements & 14 JPT & 30 JPT & 60 JPT & 90 JPT \\
\hline AC2,5 & $19 a$ & $48 a$ & $60 a$ & $73 a$ \\
AC5 & $18 a$ & $33 a$ & $36 a$ & $42 a$ \\
LU5 & $20 a$ & $25 a$ & $28 a$ & $46 a$ \\
LU2,5 & $17 a$ & $43 a$ & $70 a$ & $88 a$ \\
TM & $73 b$ & $103 b$ & $134 b$ & $154 b$ \\
\hline
\end{tabular}

JPT -jours post traitement ; TM -Témoin ; AC2,5 -Action $80 \circledast$ à 2,5 kg/ha ; AC5 -Action 80® à $5 \mathrm{~kg} / \mathrm{ha} ;$ LU2,5 -Lumax® à 2,5 L /ha ; LU2,5 -Lumax® à 5 L/ha 
Kone et al., J. Appl. Biosci. 2020 Efficacité biologique des herbicides ACTION 80® et LUMAX® dans le contrôle des adventices dans les pépinières sèches de Tectona grandis à Sangoué en Côte d'Ivoire

Tableau 6: Évolution du taux d'enherbement en fonction des doses d'herbicide (en \%)

\begin{tabular}{l|l|l|l|l|l|l}
\hline Traitement. & 2 semaines & $\mathbf{3}$ semaines & $\mathbf{2}$ mois & $\mathbf{2 , 5}$ mois & $\mathbf{3}$ mois & $\mathbf{4 , 5}$ mois \\
\hline AC5 & 24,66 & 32,04 & 26,87 & 28 & 27,27 & 33,14 \\
AC2,5 & 26,03 & 46,60 & 44,78 & 48 & 47,40 & $\mathbf{5 4 , 8 6}$ \\
LU5 & 27,40 & 24,27 & 20,90 & 28 & 29,87 & 29,71 \\
LU2,5 & 23,29 & 41,75 & $\mathbf{5 2 , 2 4}$ & $\mathbf{5 6 , 6 7}$ & $\mathbf{5 7 , 1 4}$ & $\mathbf{5 2 , 5 7}$ \\
\hline
\end{tabular}

Tableau 7 : Nombre moyen de plantules de Teck germés en fonction du temps

\begin{tabular}{l|c|c|c|c|c}
\hline Traitements & $\mathbf{1 4} \mathbf{J P T}$ & $\mathbf{3 0} \mathbf{J P T}$ & $\mathbf{6 0} \mathbf{J P T}$ & $\mathbf{7 5} \mathbf{J P T}$ & $\mathbf{9 0} \mathbf{J P T}$ \\
\hline AC2,5 & $4,5 \mathrm{c}$ & $15,75 \mathrm{a}$ & $12,5 \mathrm{ab}$ & $12,25 \mathrm{a}$ & $11 \mathrm{ac}$ \\
AC5 & $1,5 \mathrm{ab}$ & $16 \mathrm{a}$ & $29,5 \mathrm{c}$ & $29,25 \mathrm{~b}$ & $26,5 \mathrm{~b}$ \\
LU2,5 & 0 & $16,5 \mathrm{a}$ & $20 \mathrm{ac}$ & $19,5 \mathrm{ab}$ & $18,25 \mathrm{ab}$ \\
LU5 & $0,5 \mathrm{a}$ & 0 & 0 & 0 & 0 \\
TM & $4 \mathrm{bc}$ & $7,5 \mathrm{ab}$ & $16 \mathrm{a}$ & $15,5 \mathrm{a}$ & $16,25 \mathrm{ab}$ \\
\hline
\end{tabular}

\section{DISCUSSION}

Cette étude a montré que l'efficacité des herbicides utilisés dépend de la composition des substances actives et des doses de ceux-ci. Kouamé (2014) a montré dans ses travaux dans le district de Yamoussoukro que l'efficacité des herbicides qu'il a utilisés était fonction de la composition des substances actives et de la flore en présence. De façon générale, l'utilisation des herbicides dans cette étude à permis de réduire l'enherbement des parcelles. En effet, de nombreux essais en situation réelle réalisés par le Centre Technique Forestier Tropical avec le Diuron, le Trichlopyr, le 2,4-D, du Pichlorame et le Dalapan (SODEFOR, 1998) ont montré que le Diuron (Action $80 \otimes)$ est très efficace sur les dicotylédones telles que les plantes de la famille des Asteraceae et des Solanaceae. Cependant, son efficacité selon les rapports d'essai est peu marquée sur les espèces appartenant à la famille des Poaceae. Cette tendance sélective du Diuron a été observée au cours des différents essais de la présente étude. Plusieurs travaux ont montré que certaines espèces peuvent ne pas être sensibles à un herbicide dans une flore de mauvaises herbes données (FAO, 1988; Kouamé, 2014). Selon le second auteur, l'association PropanilTrichlopyr ne contrôle pas Echinochloa crus-galli et Leptochloa caerulescens. Ceci, vient corroborer le fait

\section{CONCLUSION}

Les deux herbicides "Action $80 ®$ » et "Lumax® SE » ont permis de contrôler les Dicotylédones, Graminées, Cypéracées et de maintenir les parcelles traitées à un niveau d'enherbement inférieur à celui du seuil de nuisibilité. Selon les produits et doses utilisés, les qu'il peut exister l'aptitude à la tolérante vis à vis d'une matière active par certaines espèces. Ainsi, pour résoudre cette impasse, il serait judicieux d'utiliser des produits appartenant à des familles chimiques autres que celle déjà utilisée. L'action des différentes doses des herbicides testés est perceptible à partir de 60 JAT. Cette efficacité se relève jusqu'à la fin de l'expérimentation pour toutes les doses à l'exception de AC1. Cette situation pourrait s'expliquer par le fait que les herbicides ont une longue rémanence et un effet de préémergence. Leur utilisation permet de contrôler la levée des adventices et de retarder la germination des graines enfouies dans le sol. Cela se traduit par le long temps de rémanence observé avec les différentes matières actives. De plus, le recouvrement des parcelles traitées avec les plus fortes doses des produits est faible comparativement à celui des parcelles traitées à dose faible. Ces résultats montrent que l'efficacité des deux herbicides augmente avec la dose. La germination quasi nulle des semences de teck sur les parcelles du traitement LU5 pourrait s'expliquer par la forte dose du produit et de la forte rémanence de cet herbicide dans le sol. Cette inhibition de la germination des semences résulte du mode d'application de cet herbicide.

efficacités observées étaient différentes d'une dose à une autre. Les doses AC5 et LU5 ont montré les meilleures efficacités et ont permis d'avoir un bon contrôle des adventices. Les différentes doses des produits ont pu maintenir les parcelles exemptes 
d'adventices jusqu'à un mois après traitement. L'étude montre que l'efficacité des différentes doses des produits testés s'est révélée bonne pour tous les traitements pendant un mois après l'application. De

\section{BIBLIOGRAPHIE}

Dore T., Le bail M., Martin P., Ney B. et Roger-estrade J., 2006. L'agronomie aujourd'hui.Versailles: Présentation sur le site des éditions Quae, 367 p.

FAO, 1988. La lutte raisonnée contre les mauvaises herbes. Manuel de l'instructeur. (Collection FAO : Formation, $\left.n^{\circ} 12\right) .159 p$.

Hamel, 1986. Une technique de lutte chimique contre Eupatorium odoratum (L.) pour les replantations de palmier à huile. Oléag. 41 (6) : 263-267.

J.P. Caussanel, (1989). Nuisibilité et seuil de nuisibilité des mauvaises herbes dans une culture annuelle: relation de concurrence bispécifique. Agronomie, vol. 9, pp 219-240.

Koch W., Beshir M. E. R. (1982). Unterladstatter, Crop losses due to weeds. Improving weed management. FAO Plant Production and Protection Paper. Rome 6-10 septembre, 1982, 44. pp. 153-165,

Kouamé K. F., 2014.- Contraintes liées à la production rizicole, étude de la flore et de la végétation adventices de la riziculture dans la région du Bélier (Centre de la Côte d'Ivoire). Thèse de l'Université Félix Houphouët Boigny, Abidjan, Côte d'Ivoire, $145 p+$ annexes

Ndabalishye I., 1995. Agriculture vivrière ouestafricaine à travers le cas de la Côte d'Ivoire. Monographie, IDESSA, Bouaké, Côte d'Ivoire, $384 \mathrm{p}$.

Orkwor G. C., (1983). Problems of weed control in mixed cropping systems in the least developed countries (LDCs). C.R. llème Conf. Bis. SOAM/WAWSS. Abidjan : 95-13.

SODEFOR, 1998. Acte de l'atelier sur les reboisements. Document interne, $164 \mathrm{p}$.

Yapi A F., 2017. Mauvaises herbes majeures et itinéraires techniques de désherbage des cultures vivrières de la Région de la Mé au sud-est de la Côte d'Ivoire : Cas de la banane plantain et du manioc. Thèse de doctorat, UFR Biosciences Université Félix Houphouët Boigny Côte d'Ivoire ; 143 p. plus, la persistance des traitements varie entre quatre (4) et (6) mois. La dose minimale efficace pour l'herbicide Lumax® est de $3 \mathrm{l} / \mathrm{ha}$. Celle de Action $80 ®$ est de l'ordre de $4 \mathrm{~kg} / \mathrm{ha}$. 